\title{
Algorithms for diffusion and convection models implemented using open source libraries
}

\author{
E. Mas-Hernández $^{\text {a b (D., E. A. Mancilla-Barría }}{ }^{\text {a }}$, M. de la Torre-Sanzana ${ }^{a}$ and S. Berres $^{\text {b }}$

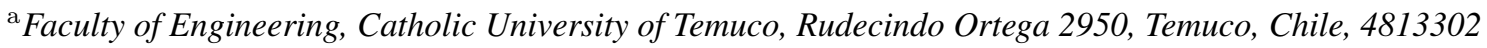 \\ ${ }^{\mathrm{b}}$ Bioproducts and Advanced Materials Research Nucleus (BioMA), Catholic University of Temuco, \\ Rudecindo Ortega 2950, Teтuco, Chile, 4813302 \\ Email: elimher@gmail.com
}

\begin{abstract}
There are several phenomena where the description by mathematical models is in terms of differential equations. Among these phenomena, we can find (1) the diffusion of pollution particles, (2) the growth of metal nanoparticles, and (3) the displacement of foam within porous media. In this paper we present some solutions for models that depict the two first processes of interest mentioned above, which were programmed using libraries for Python ${ }^{\mathrm{TM}}$, and explore the possibility of applying the programming framework to the third application.

The diffusion of air pollutants, specially particulate matter, can be modelled with an advection-diffusion equation. This equation makes it possible to consider the phenomena that describe the change in concentration of particulate matter $\left(\mathrm{PM}_{10}\right.$ and $\left.\mathrm{PM}_{2.5}\right)$ with time and also the effects of wind and rain. The chosen method to tackle the solution for the advection-diffusion equation is the finite-volume method, and its respective algorithm makes use of a triangular grid.

On the other hand, the growth of copper nanoparticles on silicon surfaces can be approached from different perspectives. Until now we have explored curve fitting to obtain equations that approximate experimental data. In addition, the Hamilton-Jacobi equation has been also used to describe this process. Both, curve fitting and the solution of Hamilton-Jacobi equation have been implemented in Python.

Finally, the flow of a foam front inside a porous medium can be described using a simplified model for bubble films known as the pressure-driven growth $\mathrm{m}$ odel. In this application, different formulations can be used as well. In particular, an Eulerian model can make use of the so-called Eikonal equation to address its solution using triangular meshes.

Implementations of the algorithms to solve the models for the applications of interest use libraries for Python, mainly NumPy, OpenMesh ${ }^{\odot}$, Matplotlib, pandas, and scikit-image.
\end{abstract}

Keywords: Open software, differential equations, contaminants diffusion, copper nanoparticles, foams 
E. Mas-Hernández et al., Algorithms for diffusion and convection models ...

\section{INTRODUCTION}

Several mathematical models that describe physical phenomena can be expressed by differential equations [Cha et al. (2000), Roberts (2010)] that generally require numerical methods to determine their solutions, in particular if analytic solution are not available. In turn, these numerical methods entail algorithms that are coded using various kinds of programs.

Balance principles for the derivation of models such as the conservation of mass, energy or momentum lead to equations considered within the theory of conservation laws, which can be set in integral or differential forms [LeVeque (1992)].

Furthermore, the phenomena of interest in this study, namely, (1) the diffusion of pollutants, (2) the growth of metal nano particles and (3) the displacement of a foam front, can be laid out with an advection-diffusion equation, the Hamilton-Jacobi equation and the Eikonal equation, respectively [LeVeque (1992), Osher \& Fedkiw (2003), Sethian (1996)].

Among the different numerical approaches that can be used to deal with conservation laws the finite volume method is a suitable alternative [Eymard et al. (2000)]. We take advantage of the possibility of using triangular meshes that this method allows. In addition finite differences can also be employed for the solution of the Hamilton-Jacobi equation with level set methods [Li et al. (2010)].

In the phase of implementation of the modeling process we choose to use, create and reuse open source software, specifically within the Python ${ }^{\mathrm{TM}}$ ecosystem, manly by integration of the following libraries: NumPy [Harris et al. (2020)], OpenMesh ${ }^{\odot}$, Matplotlib [Hunter (2007)], pandas [McKinney (2010)] and scikit-image [van der Walt et al. (2014)]. In this way we can make the documentation, algorithms and codes available to anyone interested on developing this information starting from what we already obtained.

The structure of the paper is as follows: in Section 2 background, theory and results about the modeling of air pollution is presented. Section 3 deals with modeling the growth of copper nanoparticles. Section 4 is about the displacement of a foam front in porous media. Section 5 presents conclusions.

\section{DIFFUSION MODEL FOR AIR POLLUTION}

Particulate matter. Nowadays air pollution by particulate matter is one of the most relevant environmental and health concerns, mostly in urban areas. Particulate matter is defined as contaminants found in the air in the form of solid particles and liquid droplets which contain acids, organic chemicals, metals, and soil or dust particles [Anderson et al. (2012)]; they are classified according to their size as $\mathrm{PM}_{10}$ : with $10 \mu \mathrm{m}$ diameters, and $\mathrm{PM}_{2.5}$ : diameter of $2.5 \mu \mathrm{m}$ and smaller [United States Environmental Protection Agency (n.d.)].

According to Newell et al., low and middle-income countries suffer this problem due to the use of wasteful devices and fuels. In the case of the southern region of Chile, the principal source for household heating is firewood, which leads to emissions of particulate matter, primarily during the winter season, additional to emissions from automobiles and industry. The Chilean Environmental Agency collects data and provides information on air quality [Ministerio del Medio Ambiente (n.d.)]. In the particular case of the Temuco-Padre las Casas conurbation, in the Aruacania region, there are only three fixed monitoring stations that provide air quality data, which may not be representative. Therefore, we are interested in simulating how particulate matter moves through the local urban area.

Model. The proposed model considers the use of the advection-diffusion equation that in turn takes into account Fick's law to describe the transport of particulate matter through the air. It also includes terms for functions corresponding to emissions, and settling. This model is presented in Equation (1).

$$
\frac{\partial u}{\partial t}(x, y, t)+\nabla \cdot(V u)=\nabla \cdot(\mathbb{D} \nabla u)+E(x, y, t)-k u
$$

with initial condition: $u(x, y, 0)=u_{0}(x, y)$; where, $u$ is the pollutant concentration, $t$ is time, $x$ and $y$ are spatial coordinates for a two-dimensional domain, $V$ represents the wind velocity, $\mathbb{D}$ stands for the diffusion coefficient, $E$ is a function for emissions, and $k$ is a settling function. 
E. Mas-Hernández et al., Algorithms for diffusion and convection models ...

Integrating Equation (1) with respect to the control area, $C$, the following expression is obtained:

$$
\begin{aligned}
\int_{\Delta t} \int_{C} \frac{\partial u}{\partial t}(x, y, t) \mathrm{d} A \mathrm{~d} t=\int_{\Delta t} \int_{C} \nabla \cdot(\mathbb{D} \nabla u) \mathrm{d} A \mathrm{~d} t- & \int_{\Delta t} \int_{C} \nabla \cdot(V u) \mathrm{d} A \mathrm{~d} t \\
& \quad+\int_{\Delta t} \int_{C} E(u) \mathrm{d} A \mathrm{~d} t-\int_{\Delta t} \int_{C} k u \mathrm{~d} A \mathrm{~d} t .
\end{aligned}
$$

The physical domain consists on a square polygon $\left(\Omega \in \mathbb{R}^{2}\right)$ that covers the urban area of interest, which considers only the horizontal effects of advection and diffusion, without taking into account the axis $z$ for height.

Equation (2) is discretized to be solved via the finite volume method. Boundary conditions are considered as periodic, assuming a continuity on the mesh edges. A fine mesh is generated using a Delaunay triangulation in OpenMesh; information about the cell barycenters, cell areas, edge centers, edge lengths, and unit normal vectors to the cells, is obtained, as well as the connectivity information between the cells. In order to obtain the concentration values on the mesh cells, first the parameters and initial concentration are given, and the calculations are performed in the overall mesh (vertices and cells) until certain time value. Later on, the advective and diffusive fluxes are computed using explicit and implicit methods.

In addition to numerical simulations, an analytical solution has also been obtained to benchmark the results. Implementations of the numerical solution on Python can be found at the following repository: https: //github.com/guayiyo89/AdvectionDiffusion.

Results. The simulations allow obtaining data on the concentration of particulate matter. Figures 1 and 2 show the solution on a fine triangular mesh that indicate the concentration of particulate matter.

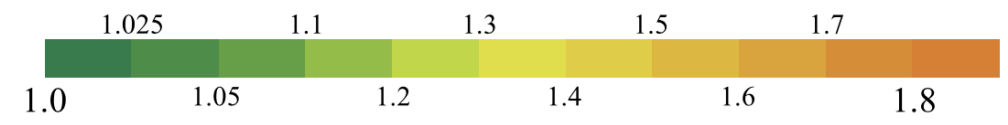

(a)

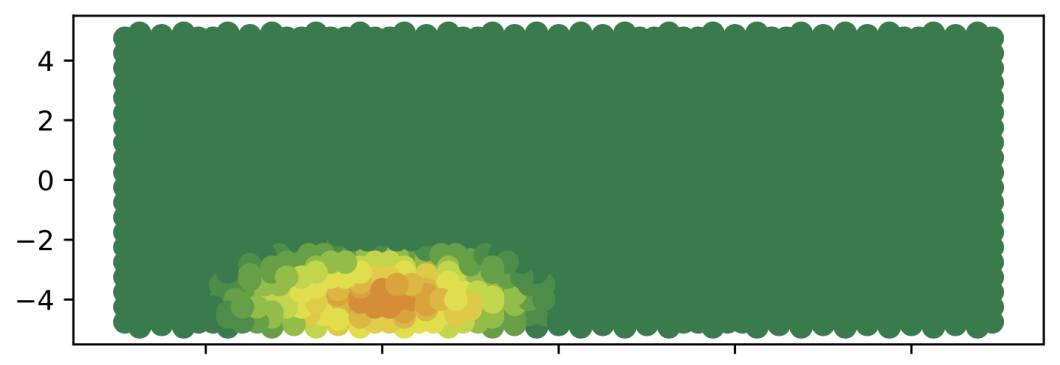

(b)

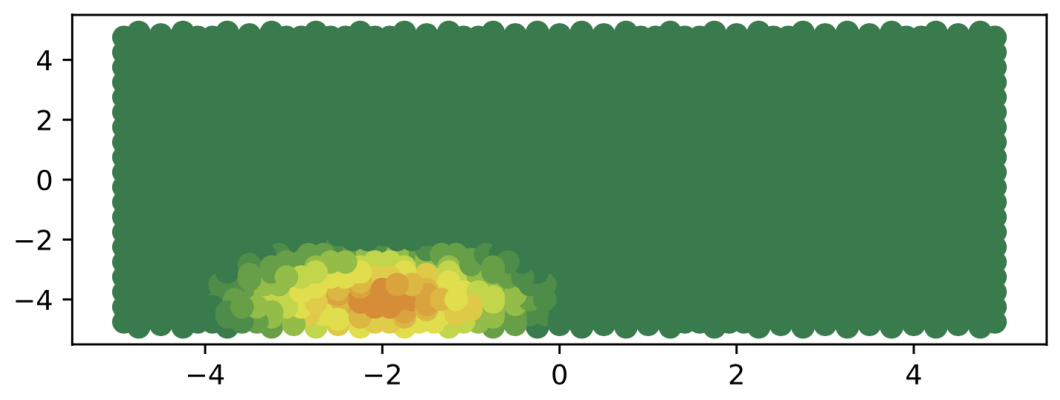

(c)

Figure 1. Particulate matter concentration at initial time $t=0$. (a) Color scale indicating concentration $u$, (b) Numerical solution, (c) Analytical solution. 
E. Mas-Hernández et al., Algorithms for diffusion and convection models ...

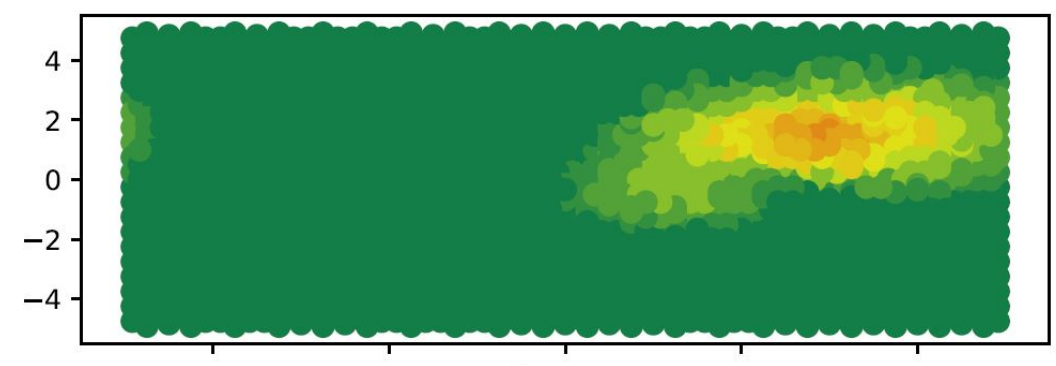

(a)

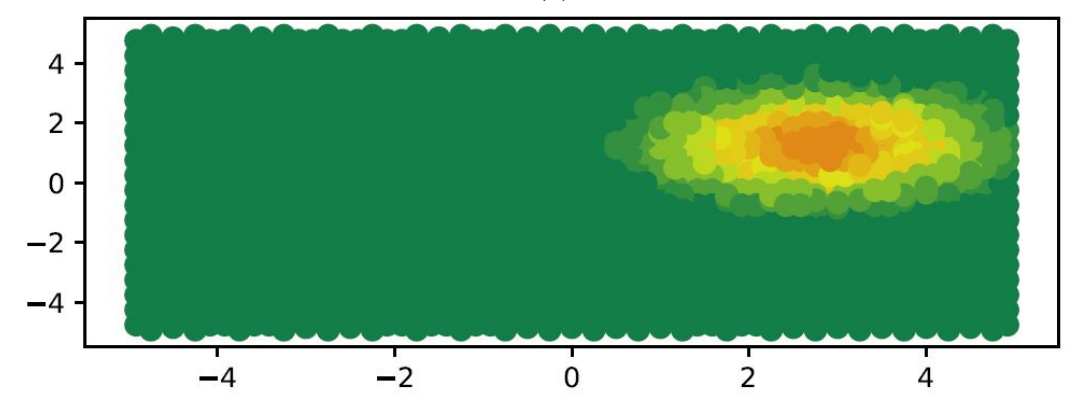

(b)

Figure 2. Particulate matter concentration at time $t=3$. (a) Numerical solution, (b) Analytical solution.

It can be observed in Figure 1 that both solutions start with the same particulate matter concentration, but with time the results are slightly different as shown in Figure 2

\section{GROWTH OF COPPER NANOPARTICLES}

Mathematical modeling for the growth of metal particles own its importance to the different potential applications for the implicated processes where this phenomenon is presented, foremost for the manufacturing of electronic devices [Oskam et al. (1998), Ghosh (2019)]. One specific case we are interested in is the evolution of copper nanoparticles deposited on silicon surfaces [Recio et al. (2014)].

The approach we have explored consists of two main aspects. One is determining models that describe experimental data using a numerical scheme for image processing. This gives information on the number of particles and the area of the silicon surface they cover . The other considers using a Hamilton-Jacobi equation coupled with a diffusion equation to describe the process. This is based on previous works such as the contribution of Wei that set the copper deposition model as a reaction-diffusion equation and use level set methods for the formulation of motion of boundaries [Osher \& Fedkiw (2003)].

Models. A simple equation to describe the growth of copper nanoparticles on a silicon surface was derived fitting a function to experimental data. It is important to note that to be able to perform this step a previous data processing stage was needed. The inputs for data processing consist of images obtained through atomic force microscopy (AFM) of silicon substrates with deposited copper nanoparticles through electrodeposition, using aqueous solutions of $\mathrm{CuSO}_{4}$; the current density was fixed at $100 \mu \mathrm{A} / \mathrm{cm}^{2}$. Image processing was done using a code implemented on Python. The outputs are data for the particle size $(Y)$ versus time $(t)$ from where the function presented in Equation (3) is obtained.

$$
Y=\frac{4}{125} t^{2}-\frac{21}{25} t+\frac{122}{5}
$$

On the other hand, it is possible to consider this phenomenon as the propagation of the copper surface. Therefore, a model that can depict this case uses a Hamilton-Jacobi equation

$$
v_{t}+\vec{v} \cdot \nabla v=0
$$

where $v=v(x, y, t)$ is the propagation velocity. 
E. Mas-Hernández et al., Algorithms for diffusion and convection models ...

Substituting the velocity vector, $\vec{v}$, for the propagation of copper particles in Equation (4), we obtain:

$$
v_{t}=\frac{F c^{r}}{v^{*}}|\nabla v|
$$

where $c$ is concentration of copper ions, $v^{*}$ is the level set function inside the interface of interest, $F$ and $r$ are parameters typical of the application and they are defined in Wei (2011).

The approach to solve Equation (5) is the formulation known as distance regularized level set evolution (DRLSE) [Li et al. (2010)], which avoids the reinitialization step when using level set methods and also allows using finite differences as an alternative to the finite volume method. Implementations of algorithms on Python can be found at https://github. com/mauriciodelatorre

Results. The growth of copper seeds specifically selected was analyzed. Figure 3 shows a nanoparticle with a simulated boundary at $30 \mathrm{~s}$.

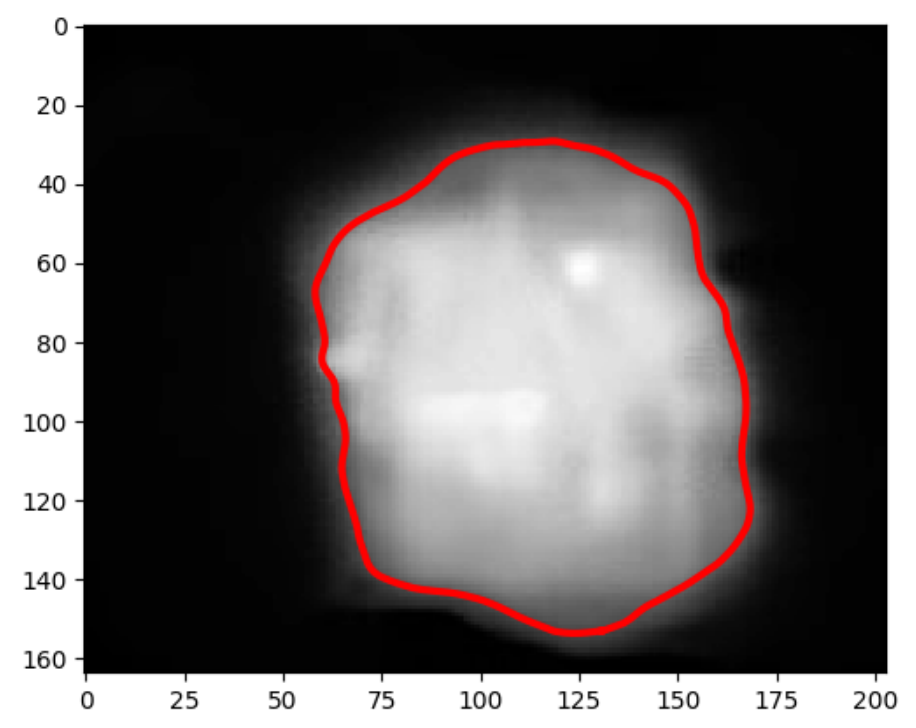

Figure 3. Level set function evolution (red contour) for the growth of a copper seed at time $t=30 \mathrm{~s}$.

We therefore verify that the simulations are able to track the motion of the copper seed surfaces.

\section{FOAM FRONT PROPAGATION}

The displacement of a foam front in porous media can be modeled using a system of hyperbolic differential equations obtained when Darcy's law is applied to the interstitial velocity of the fluid, the so-called Pressuredriven growth model [Grassia et al. (2014)]. The model has been approached employing both Lagrangian and Eulerian schemes. However, one pending method to study is the formulation as a boundary value problem that leads to a formulation that is equivalent to the Eikonal equation [Sethian (1996)].

Model. The model for the foam front propagation set as a system of Eikonal equations for a two-dimensional domain with coordinates $(x, y)$ on a irregular grid can be set by

$$
\begin{aligned}
& \|\nabla T\|=v \\
& \frac{\nabla T}{\|\nabla T\|}=\nabla S,
\end{aligned}
$$


where $T$ is the time solution value for the mesh node or cell, $v$ is the propagation velocity, $S$ is the front path length, and $\|\cdot\|$ indicates the norm. The system in equation (6) can also be expressed as:

$$
\begin{aligned}
& H_{T}(T, S)=\|\nabla T\|-v=0 \\
& H_{S}(T, S)=\frac{\nabla T}{\|\nabla T\|}-\nabla S=0,
\end{aligned}
$$

where $H$ stands for the Hamiltonian.

Projection. The algorithms and codes for the diffusion of particulate matter and growth of copper nanoparticles will be adjusted to solve the foam front displacement model. In such a way, we can explore different methods: finite volume methods on triangular meshes, and level set methods, among others.

\section{CONCLUSIONS}

The diffusion of particulate matter within air pollution and the growth of copper nanoparticles on silicon surfaces can be modeled with an advection-diffusion equation and the Hamilton-Jacobi equation, respectively. The simulations make use of Python and some of its libraries. In both cases, results have been obtained for the concentration of particulate matter on a 2D domain, and the tracking of copper seed surfaces. We expect to adapt these codes for other applications such as the displacement of a foam front inside porous medium. Thus, we were able to implement algorithms for these models using an open source software. The codes and repositories are publicly available for anyone interested in these applications.

\section{ACKNOWLEDgement}

EMH acknowledges funding by the National Agency for Research and Development (ANID) FONDECYT POSTDOCTORATE 3200839.

\section{REFERENCES}

Anderson, J. O., Thundiyil, J. G. \& Stolbach, A. (2012), 'Clearing the Air: A Review of the Effects of Particulate Matter Air Pollution on Human Health', Journal of medical toxicology 8(2), 166-175.

Cha, P. D., Rosenberg, J. J. \& Dym, C. L. (2000), Fundamentals of Modeling and Analyzing Engineering Systems, Cambridge University Press, United States of America.

Eymard, R., Gallouët, T. \& Herbin, R. (2000), Finite Volume Methods, in 'Solution of Equation in $\mathbb{R}^{n}$ (Part 3), Techniques of Scientific Computing (Part 3)', Vol. 7 of Handbook of Numerical Analysis, Elsevier, pp. 713-1018.

Ghosh, S. (2019), 'Electroless Copper Deposition: A critical review', Thin Solid Films 669, 641-658.

Grassia, P., Mas-Hernández, E., Shokri, N., Cox, S. J., Mishuris, G. \& Rossen, W. R. (2014), 'Analysis of a Model for Foam Improved Oil Recovery', Journal of Fluid Mechanics 751, 346-405.

Harris, C. R., Millman, K. J., van der Walt, S. J., Gommers, R., Virtanen, P., Cournapeau, D., Wieser, E., Taylor, J., Berg, S., Smith, N. J., Kern, R., Picus, M., Hoyer, S., van Kerkwijk, M. H., Brett, M., Haldane, A., del Río, J. F., Wiebe, M., Peterson, P., Gérard-Marchant, P., Sheppard, K., Reddy, T., Weckesser, W., Abbasi, H., Gohlke, C. \& Oliphant, T. E. (2020), 'Array programming with NumPy', Nature 585(7825), 357-362.

Hunter, J. D. (2007), 'Matplotlib: A 2D Graphics Environment', Computing in Science \& Engineering 9(3), 90-95.

LeVeque, R. (1992), Numerical Methods for Conservation Laws, 2 edn, Birkhäuser Verlag, Basel, Switzerland.

Li, C., Xu, C., Gui, C. \& Fox, M. D. (2010), 'Distance Regularized Level Set Evolution and Its Application to Image Segmentation', IEEE Transactions on Image Processing 19(12), 3243-3254.

McKinney, W. (2010), Data Structures for Statistical Computing in Python, in Stéfan van der Walt \& Jarrod Millman, eds, 'Proceedings of the 9th Python in Science Conference', pp. $56-61$.

Ministerio del Medio Ambiente (n.d.), 'Sistema de Información Nacional de Calidad del Aire', https: //sinca.mma.gob.cl/index.php/. Accessed: 2021-09-01.

Newell, K., Cusack, R. P., Kartsonaki, C., Chaudhary, N. \& Kurmi, O. P. (2019), Household Air Pollution and Associated Health Effects in Low and Middle Income Countries, in 'Reference Module in Biomedical Sciences', Elsevier.

Osher, S. \& Fedkiw, R. (2003), Level Set Methods and Dynamic Implicit Surfaces, Vol. 153, Springer-Verlag New York Inc., New York.

Oskam, G., Long, J., Natarajan, A. \& Searson, P. (1998), 'Electrochemical deposition of metals onto silicon', Journal of Physics D: Applied Physics 31(16), 1927. 
E. Mas-Hernández et al., Algorithms for diffusion and convection models ...

Recio, G., Gallach, D., Manso Silvan, M., Fukami, K., Martin Palma, R. J., Castro, G. R. \& Muñoz-Noval, A. (2014), 'Photoassisted Immersion Deposition of Cu Clusters onto Porous Silicon: A Langmuir-Hill LigandLocus Model Applied to the Growth Kinetics', The Journal of Physical Chemistry C 118(27), 14905-14912.

Roberts, C. (2010), Ordinary differential equations: applications, models, and computing, CRC Press, United States of America.

Sethian, J. A. (1996), 'A fast marching level set method for monotonically advancing fronts', Proceedings of the National Academy of Sciences 93(4), 1591-1595.

United States Environmental Protection Agency (n.d.), 'Particulate Matter (PM) Basics', https://www . epa.gov/pm-pollution/particulate-matter-pm-basics. Accessed: 2021-09-01.

van der Walt, S., Schönberger, J. L., Nunez-Iglesias, J., Boulogne, F., Warner, J. D., Yager, N., Gouillart, E., Yu, T. \& the scikit-image contributors (2014), 'scikit-image: image processing in Python', PeerJ 2 , e453.

Wei, H.-C. (2011), 'Qualitative simulation of the growth of electrolessly deposited cu thin films', Mathematical Problems in Engineering 2011. 